\title{
An Education Program for Prevention of Cerebral Palsy for Pregnant Women Attending Anti-natal Clinics: A descriptive Survey
}

Auwal Abdullahi ${ }_{\text {ABCDEFF }}$ Aliyu Zakariyya ${ }_{\text {ABCDEF }}$ Muhammad Aliyu Abba ${ }_{\text {ABCDEF }}$

${ }^{1}$ Neurological Rehabilitation Unit, Department of Physiotherapy, Bayero University Kano, Nigeria. ${ }^{2}$ Department of Physiotherapy, Murtala Muhammad Specialists Hospital Kano, Nigeria

\begin{abstract}
$\mathrm{CP}$ is the commonest and a leading cause of chronic childhood disability, with profound medical, emotional, and economic consequences. The lack of knowledge among pregnant women and mothers of CP children affect their participation in the management of the condition

The aim of this study was to educate pregnant women attending antenatal clinics of Murtala Mohammed Specialist Hospital (MMSH) and Muhammad Abdullahi Wase Specialist Hospital (MAWSH) in Kano state Nigeria then, evaluate the outcome of the educational intervention programme on cerebral palsy.

The design employed in this study was a descriptive survey via series of interviews. The population of this study constituted pregnant women attending antenatal clinic of MMSH and MAWSH, participants were recruited using convenience sampling techniques

At total of 37 respondents participated in the study and baseline knowledge of cerebral palsy was obtained through an interview in the first week, education intervention on CP was administered in the second week and in the third week, the participants were interviewed to evaluate the level of their understanding post educational session on cerebral palsy.

The finding of this study showed low level of CP condition awareness among pregnant women in Kano, Nigeria, but after educational intervention in this study, their knowledge improved and participants were able to give explanations on the cause, clinical manifestation, preventive measures and management, therefore the need for educational programmes on $\mathrm{CP}$ during antenatal periods by the medical professionals or through interactive session among the women.
\end{abstract}

Key words: prevention, pregnant women, antenatal clinics, cerebral palsy

\section{Introduction}

Cerebral Palsy $(\mathrm{CP})$ can be defined as an insult to the central nervous system in the early developing brain that produces a group of non-progressive disorders of movements and postures [1].The insult leading to CP may develop either during prenatal, perinatal or postnatal period with most common etiologic factors being prematurity, ischemia, hypoxemia, hyperbilirubinemia and trauma. [2]CP has the prevalence of about 2.11 per 100 live births globally, [3][4] and manifestation of either monoplegia, diplegia, hemiplegia, triplegia, quadriplegia or double hemiplegia based on the topographic distributions of motor involvement that reflects direct area of brain affectation.[5] Other manifestations may include those problems as Mental retardation, epilepsy, bladder dysfunction, bowel dysfunction, visual, hearing disorder, sleeping disorders and orthopaedic disorders [6]

Cerebral palsy is the commonest and a leading cause of chronic childhood disability, with profound medical, emotional, and economic consequences. [7][8][9]. A child with cerebral palsy have a significant impact on the life of the family and care givers, the impact could range from physical which may include musculoskeletal pains to psychological such as stress and anxiety [10], as a result of challenge faced by the children with $\mathrm{CP}$ that requires family centered services. [6] Mortality in CP is strongly associated with both the level of functional impairment as well as associated non-motor impairments, however among the non-motor impairments intellectual; disability is found to be a strong predictor of mortality. Nonetheless, mortality risk increases with increasing number of impairments, limb function, hearing, and vision. [5] [11]

The diagnosis of cerebral palsy is basically on parent reports of delayed motor milestones, such as neck control, sitting, pulling to stand, and walking, and evaluation of posture, deep tendon reflexes, and muscle tone by the clinician. A definitive diagnosis can only be established after repeated examination because it largely depends on neurological findings such as transient dystonia which may resolve within few months and no longer present after one year of age mostly seen among infants born prematurely. [5][12] 
The definition of CP manifestation as 'permanent' is discouraging to clinicians, family or caregivers. In recent times, the perception of clinical manifestations and severity of functional impairment is that it may change over time.[5] Early intervention therapy can help achieve functional abilities that facilitate independence and improve quality of life. It includes specialized instruction and related rehabilitation services which minimize the disabling effects of cerebral palsy and promote optimal development over time. [1][9] Appropriate planning is necessary to improve their quality of life (QoL) in different domains.[6] The management of $\mathrm{CP}$ is now taking a new dimension; it involves participation of family in the early intervention [12], thereby producing effective preventative and rehabilitative measures especially during perinatal period [13].

The Participation of family in the management of children with cerebral palsy positively influences the outcome of rehabilitation due to the huge supportive care they offer to the patients, thus parents of children with cerebral palsy must have knowledge about the disease for its effective management. [9] The knowledge of the disease can be passed on to the parents through an educational intervention method from parents to parents, [14] or medical practitioners to parents [15], which in turn significantly improves parents/ caregivers' overall knowledge of cause, prognosis, and the necessary intervention early enough to prevent secondary complications. [1]

Studies document the lack of adequate knowledge of parents with cerebral palsy [1][9][16] and pregnant women with non $\mathrm{CP}$ children, despite the significance of their involvement in the management of cerebral palsy. [17] Hence, necessitating for educational intervention [16][17]. The awareness of pregnant women on cerebral palsy may contribute to them on the understanding for the necessary medical care during the period of pregnancy [17].The aim of this study is to educate pregnant women attending antenatal clinics Kano State, Nigeria on cerebral palsy and to evaluate the post educational intervention knowledge of $\mathrm{CP}$ among the participants.

\section{Material and methods}

The study involved 336 professionally active paramedics of ambulance emergency teams employed in the randomly chosen medical facilities in Poland. The research was conducted using a diagnostic survey based on two questionnaires, i.e. the orientation to life questionnaire measuring the sense of coherence (SOC-29) and the anonymous questionnaire designed by the author.

\section{Results}

There was a statistically significant correlation between the global sense of coherence, comprehensibility, manageability and meaningfulness versus opinions regarding the quality of emergency services related to the number of places of employment. Paramedics working in one medical facility show statistically significantly higher mean levels of the sense of comprehensibility, manageability and meaningfulness as well as the mean global sense of coherence compared to paramedics working in three or more places of employment. The same correlation was observed between the group working in two places and the group working in three or more places.

\section{Conclusions}

According to the paramedics surveyed, working in the health care entities other than the parent one does not adversely affect the quality of emergency services. The sense of coherence is found to be a personality variable differentiating paramedics in terms of the number of employment places.

Key words: paramedics, quality of medical services, number of places of employment, SOC

The definition of CP manifestation as 'permanent' is discouraging to clinicians, family or caregivers. In recent times, the perception of clinical manifestations and severity of functional impairment is that it may change over time.[5] Early intervention therapy can help achieve functional abilities that facilitate independence and improve quality of life. It includes specialized instruction and related rehabilitation services which minimize the disabling effects of cerebral palsy and promote optimal development over time. [1][9] Appropriate planning is necessary to improve their quality of life (QoL) in different domains.[6] The management of $\mathrm{CP}$ is now taking a new dimension; it involves participation of family in the early intervention [12], thereby producing effective preventative and 
rehabilitative measures especially during perinatal period [13].

The Participation of family in the management of children with cerebral palsy positively influences the outcome of rehabilitation due to the huge supportive care they offer to the patients, thus parents of children with cerebral palsy must have knowledge about the disease for its effective management. [9] The knowledge of the disease can be passed on to the parents through an educational intervention method from parents to parents, [14] or medical practitioners to parents [15], which in turn significantly improves parents/ caregivers' overall knowledge of cause, prognosis, and the necessary intervention early enough to prevent secondary complications. [1]

Studies document the lack of adequate knowledge of parents with cerebral palsy [1][9][16] and pregnant women with non $\mathrm{CP}$ children, despite the significance of their involvement in the management of cerebral palsy. [17] Hence, necessitating for educational intervention [16][17]. The awareness of pregnant women on cerebral palsy may contribute to them on the understanding for the necessary medical care during the period of pregnancy [17].The aim of this study is to educate pregnant women attending antenatal clinics Kano State, Nigeria on cerebral palsy and to evaluate the post educational intervention knowledge of $\mathrm{CP}$ among the participants.

\section{Methodology}

The aim of this study was to educate pregnant women attending antenatal clinics of Murtala Mohammed Specialist Hospital (MMSH) and Muhammad Abdullahi Wase Specialist Hospital (MAWSH) in Kano state Nigeria and at the same time evaluate the outcome of the educational intervention programme on cerebral palsy.

Ethical approval was obtained from Hospitals Management Board Kano State, Nigeria. The design employed in this study was a descriptive survey via series of interviews. The population of this study constituted pregnant women attending antenatal clinic of MMSH and MAWSH, participants were recruited using convenience sampling techniques. The demographic characteristics of sixty seven (67) participants' who can speak either Hausa, English or both was obtained and were screened for the study, but five (5) refused to sign the consent form, six (6) were excluded due to low score on Mini Mental State Examination scale MMSE $(<17)$ and 19 were lost to follow up. Refer to figure 1, the flow-chart summary of participants' screening.

At total of 37 respondents were able to complete the study and baseline knowledge of cerebral palsy was obtained through an interview in the first week. In the second week, the participants converged to attend an educational session on cerebral palsy about its causes, clinical manifestations, prevention and management. In the third week, the participants were interviewed to evaluate the level of their understanding post educational session on cerebral palsy. Themes were generated during the first and third interview subsequently the level of awareness was compared pre and post educational intervention. Thematic analysis and percentages were used to analyze and compare the outcome of the intervention. 


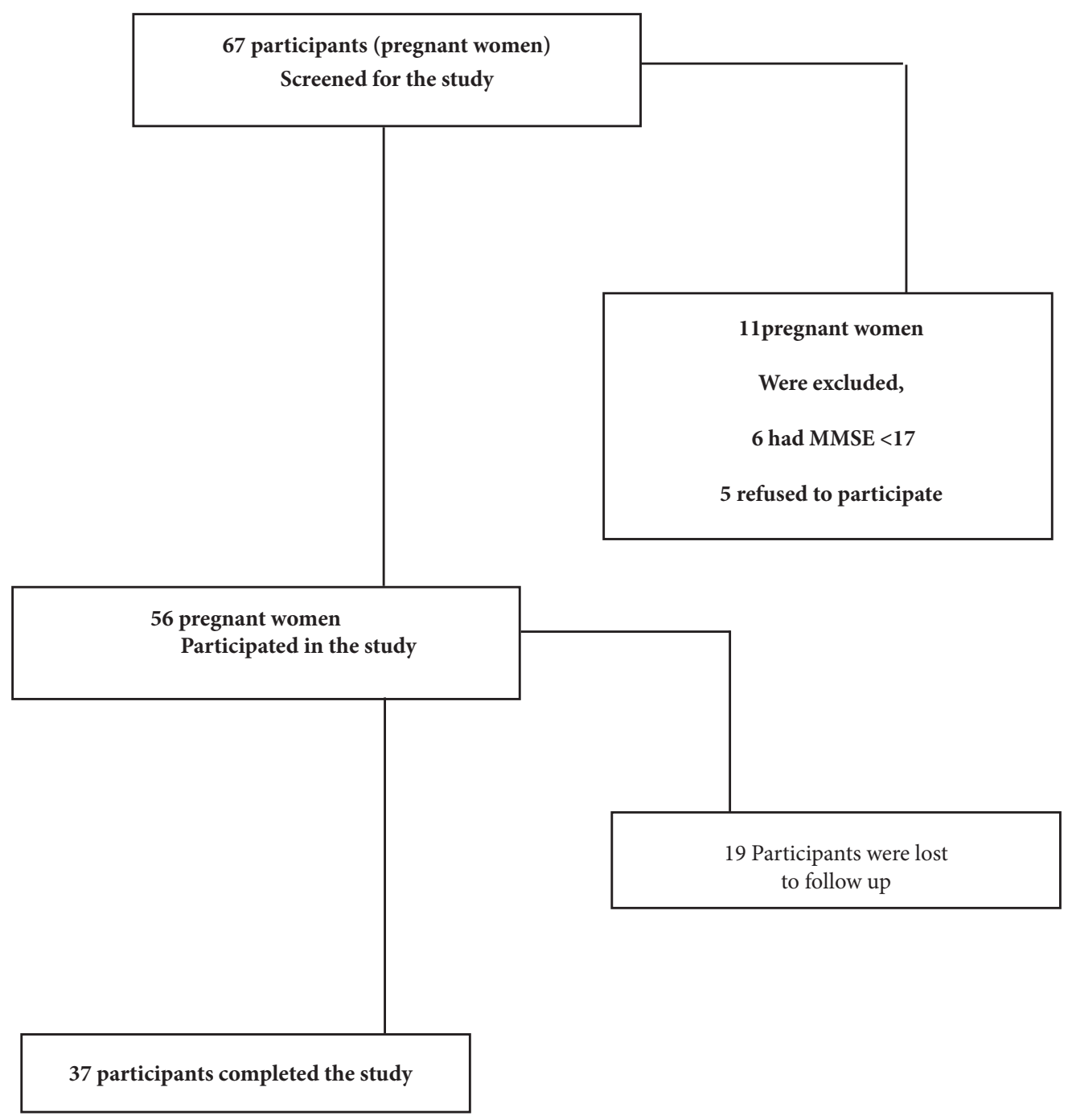

Figure 1: The Study Flowchart

\section{Results}

A total of only 37 pregnant women completed this study, the mean age of the pregnant participants was $24.5 \pm 4.187$ years. Different responses were generated from the participants during the interview pre and post educational intervention sessions. Before the awareness or educational programme to the participants on cerebral palsy, baseline responses indicated to be complete lack of knowledge of cerebral palsy expressed as follows in terms of causes 15 (40.5\%), presentation $25(67.6 \%)$, practitioners involved in management $28(75.7 \%)$, preventi- 
ve measures 20(54.1\%) and measures to be taken by parents $20(54.1 \%)$ but a significant improvement in knowledge of cerebral palsy was observed after the awareness programme with $37(100 \%)$ giving the cause of cerebral palsy as abnormalities due pregnancy. Concerning the presentation of cerebral palsy, multiple responses where expressed such as drooling of saliva in 31(83.8), lack of body control 35 (94.6\%), poor growth $30(81.1 \%)$, while on the professionals involved in the management of $\mathrm{CP}$, responses range from Paediatricians 30(81.1\%), Orthotists 33(89.2\%), Occupational therapist 25(67.6\%) to Physiotherapists 35 (94.6\%) and then on preventive measures various responses on the obtained such as Proper diet 30(81.1\%), attending clinics 34 (91.9\%), avoiding unprescribed medication $30(81.1 \%)$, avoiding early marriage 20 (54.1\%) moderate exercise, 31 $(83.8 \%)$ and Smoking cessation 33 (83.8\%) as illustrated in tables 1-5 and summarized in figure 2-3

Table 1.0 summarizes the pre and post awareness responses of the participants on cause of cerebral palsy

\begin{tabular}{ll}
\hline Themes & $\mathrm{n}(\%)$ \\
\hline & \\
Pre-awareness response & $10(27.0)$ \\
Destiny & $5(13.5)$ \\
Diseases & $15(40.5)$ \\
Not known & $8(21.6)$ \\
Pregnancy abnormalities & \\
Post-awareness response & $37(100)$ \\
Pregnancy abnormalities & $34(91.9)$ \\
Poor nutrition during pregnancy & $25(67.6)$ \\
Early marriage & $8(75.7)$ \\
Drowning of a child for some times & $23(62.2)$ \\
Jaundice & $31(83.8)$ \\
Malaria & $35(94.6)$ \\
Mrauma & $30(81.1)$ \\
\hline
\end{tabular}

$\mathrm{n}=$ number of respondents, $\%=$ percentage of the participants with the same response 
Table 2.0 below summarizes the pre and post awareness responses of the participants on the knowledge of clinical presentations of cerebral palsy

\begin{tabular}{ll}
\hline Themes & $\mathrm{n}(\%)$ \\
\hline Pre-awareness & \\
Drooling & $12(32.4)$ \\
Lack of control & $10(27.0)$ \\
Not known & $25(67.6)$ \\
Mental retardation & $4(10.8)$ \\
Poor growth & $2(5.4)$ \\
Post awareness & \\
Drooling & $31(83.8)$ \\
Lack of control & $35(94.6)$ \\
Poor growth & $30(81.1)$ \\
Difficulty with eating & $20(54.1)$ \\
Paralysis & $28(56.8)$ \\
Mental retardation & $25(67.7)$ \\
\hline
\end{tabular}

$\mathrm{n}=$ number of respondents, $\%=$ percentage of the participants with the same response

Table 3.0 below summarizes the pre and post awareness responses of the participants on the knowledge of practitioners involved in the management of cerebral palsy

\begin{tabular}{ll}
\hline Themes & $\mathrm{n}(\%)$ \\
\hline Pre-awareness & $4(10.8)$ \\
Nurses & $8(21.6)$ \\
Doctors & $28(75.7)$ \\
Not known & $2(5.4)$ \\
Physiotherapist & \\
Post awareness & $30(81.1)$ \\
Paediatricians & $33(89.2)$ \\
Orthotists & $25(67.6)$ \\
Occupational therapist & $3(8.1)$ \\
Forgotten & $35(94.6)$ \\
Physiotherapists & \\
\hline
\end{tabular}

$\mathrm{n}=$ number of respondents, $\%=$ percentage of the participants with the same response 
Table 4.0 below summarizes the pre and post awareness responses of the participants on the knowledge of preventive measures of cerebral palsy

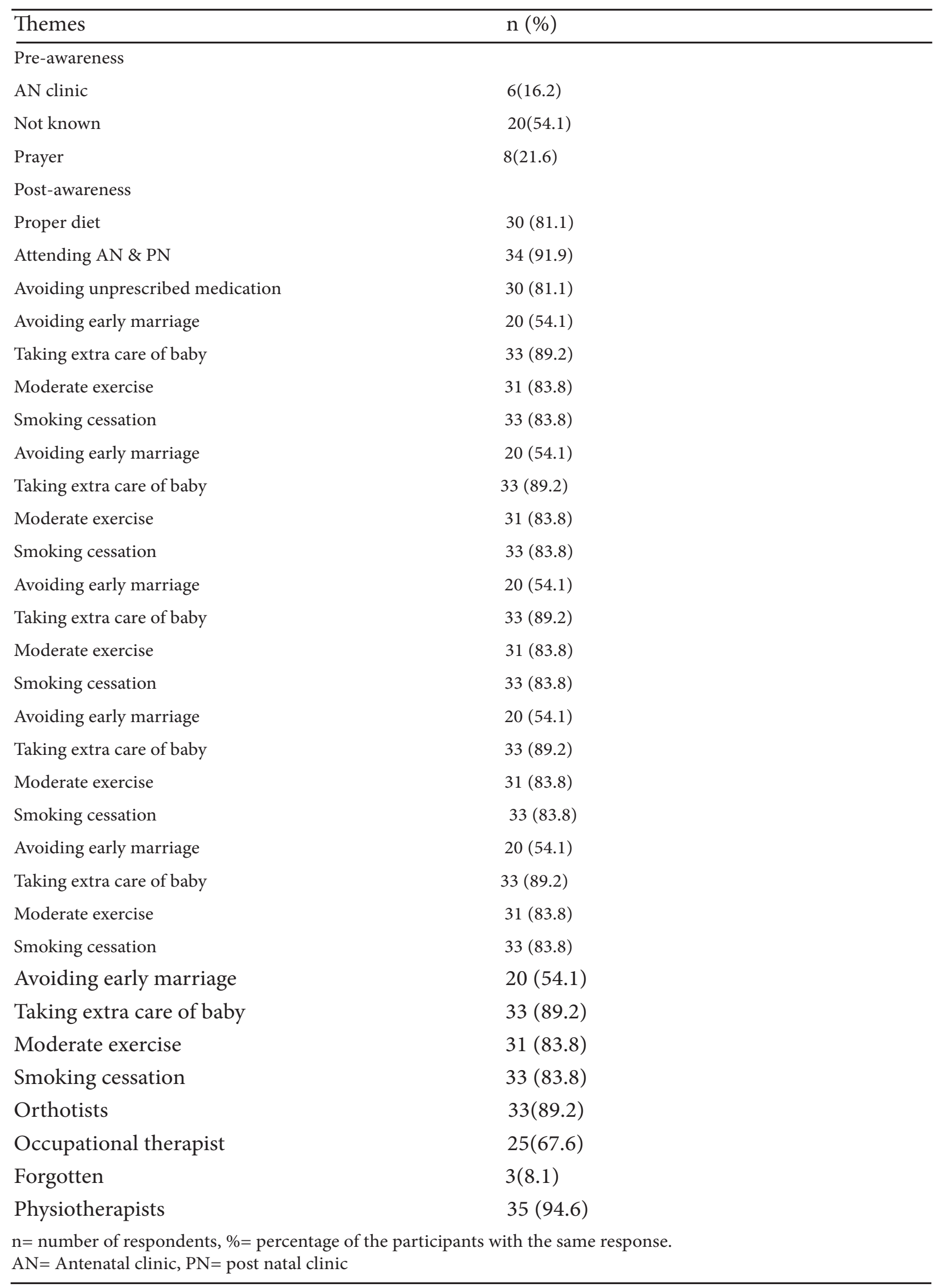


Table 5.0 below summarizes the pre and post awareness responses of the participants on the measures to be taken by mothers when child is diagnosed with cerebral palsy

\begin{tabular}{ll}
\hline Themes & $\mathrm{n}(\%)$ \\
\hline Pre-awareness & $20(54.1)$ \\
Not know & $8(21.6)$ \\
Prayer & $9(24.3)$ \\
Taking the child to doctor & \\
Post-awareness & $35(94.6)$ \\
Take the child to hospital & $2(5.4)$ \\
\hline
\end{tabular}

$\mathrm{n}=$ number of respondents, $\%$ of the participants with the same response

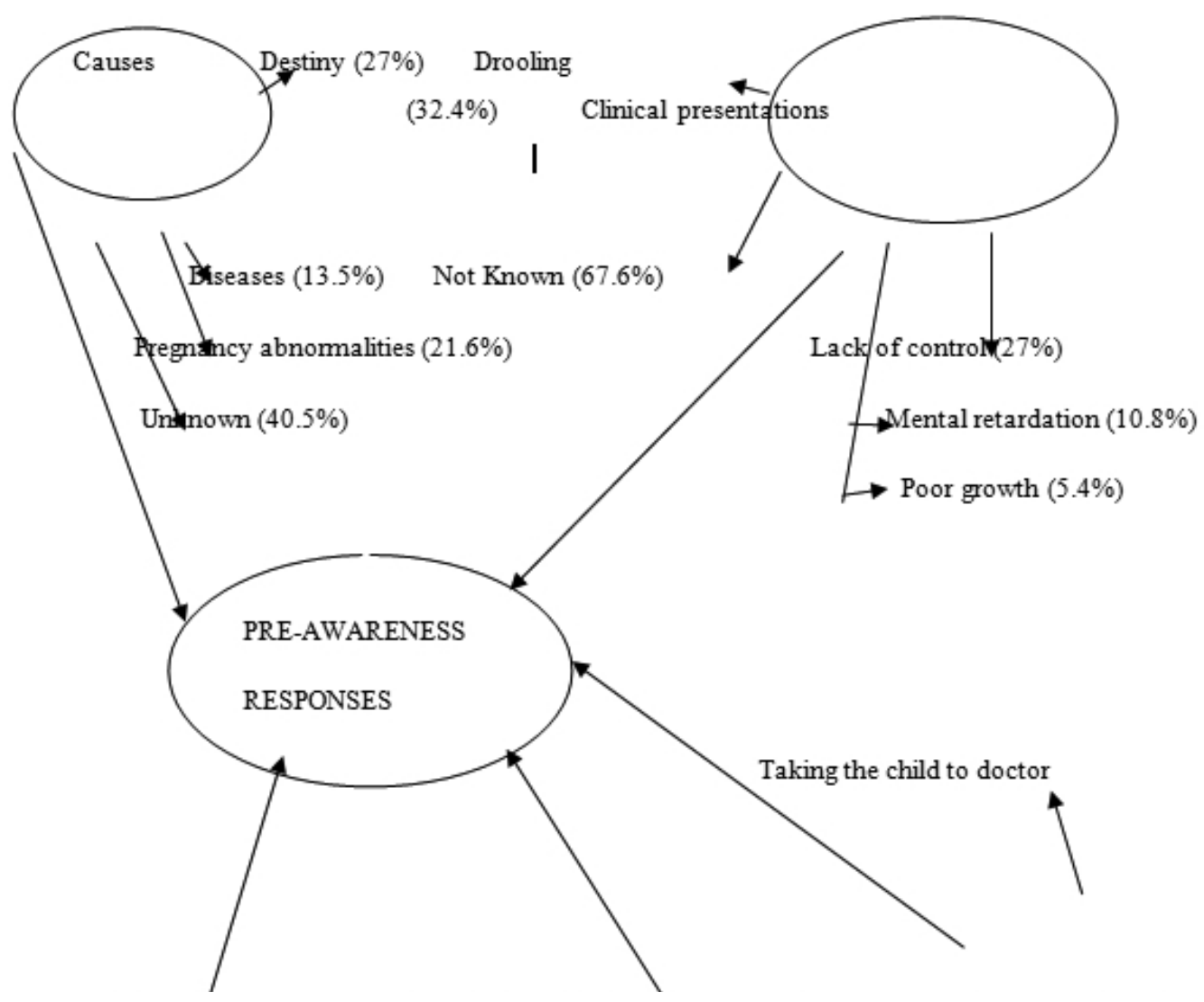


$(24.3 \%)$

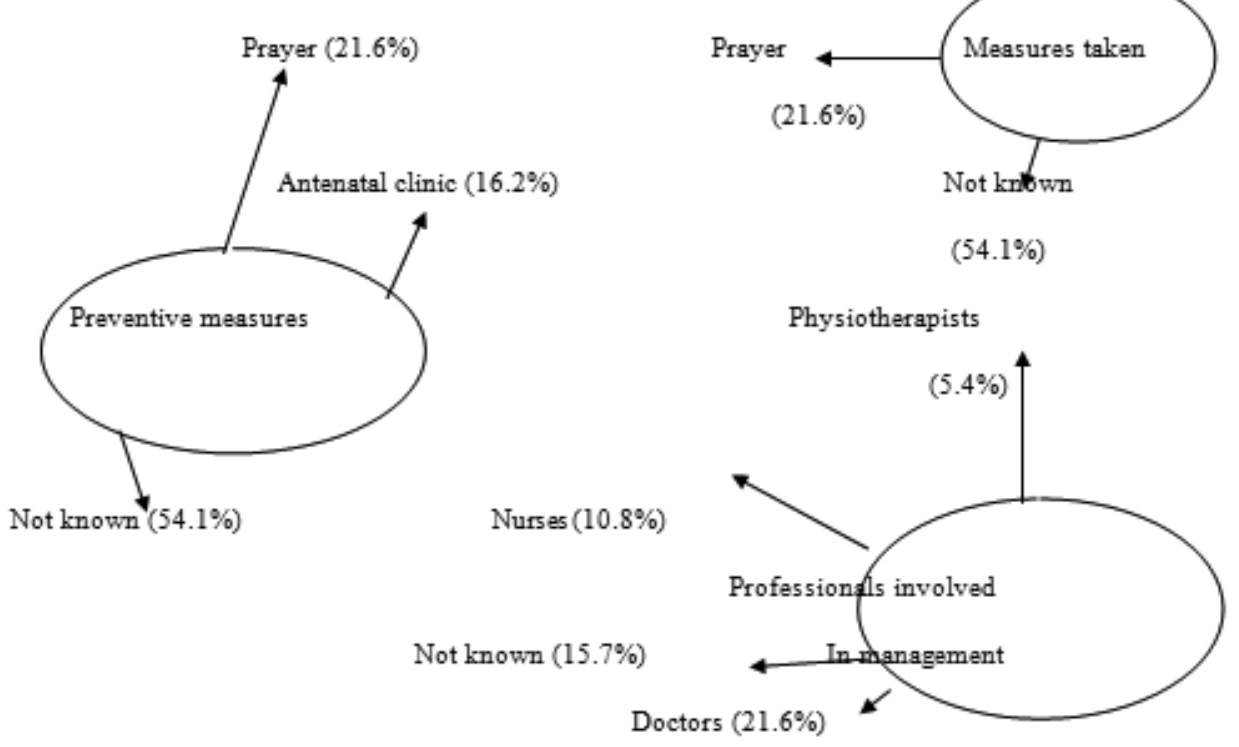

Figure 2: THEMATIC MAP OF PRE-AWARENESS RESPNSES OF PARTICIPANTS

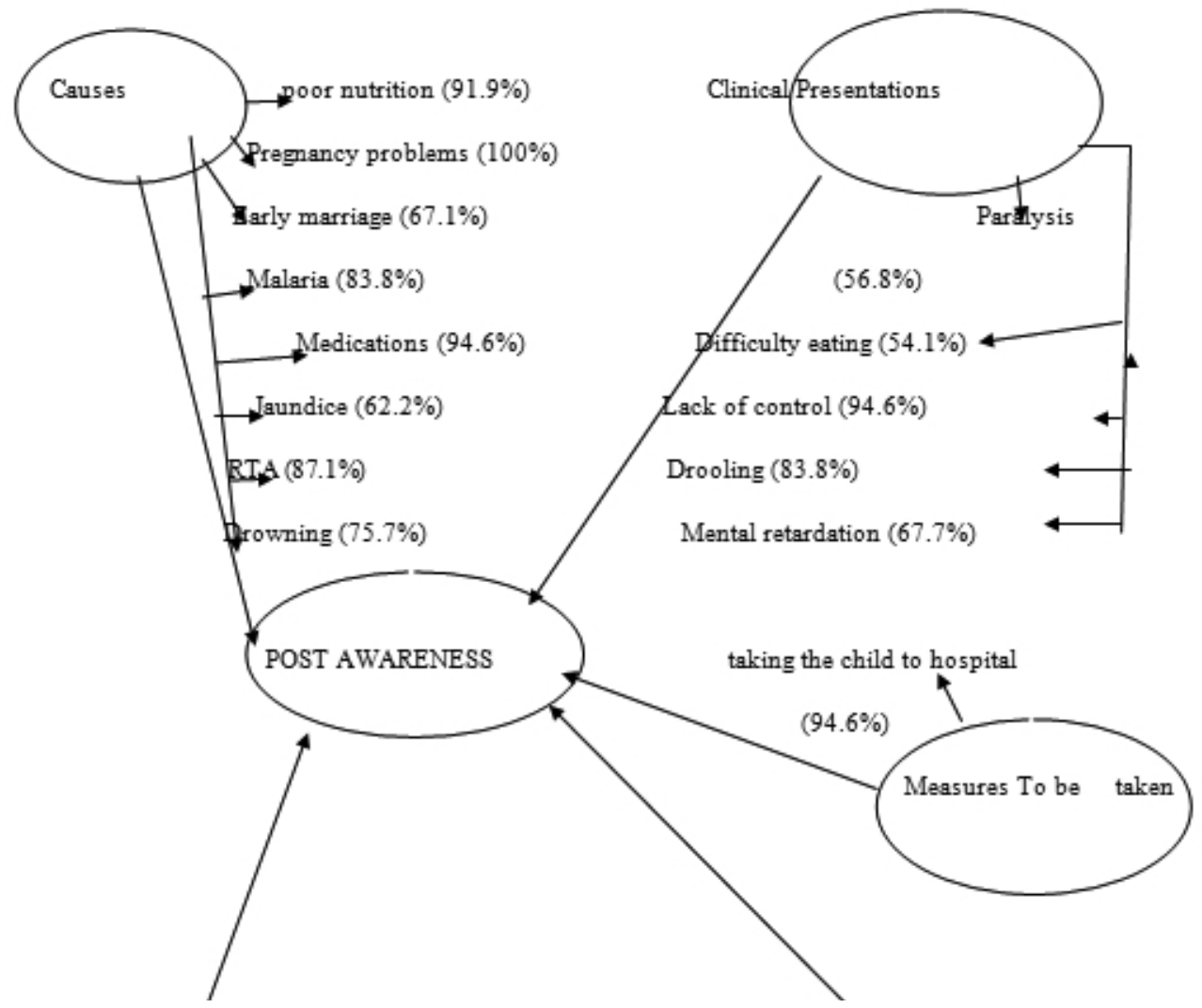




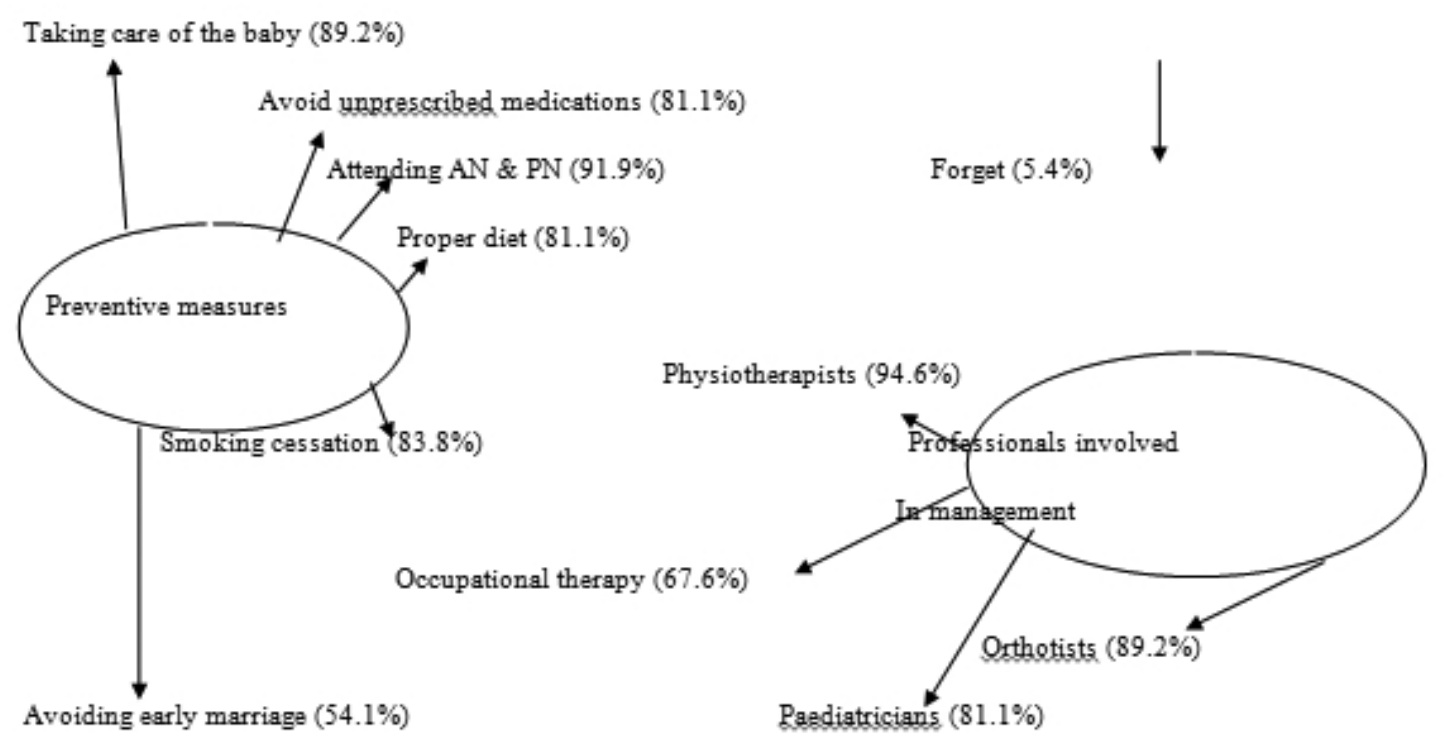

Figure 3: thematic map of post-awareness respnses of participants 


\section{DISCUSSION}

The aim of this study is to educate pregnant women attending antenatal clinics Kano State, Nigeria on cerebral palsy and to evaluate the post educational intervention knowledge of $\mathrm{CP}$ among the participants. This study reveals knowledge gap of cerebral palsy in terms of cause, clinical manifestation, prevention and management in pre and post educational intervention among pregnant women attending antenatal clinics of MMSH and MAWSH Kano state.

In about half of the participants, the pre-educational intervention awareness was about $40.5 \%$ responses were lack of the possible knowledge of the $\mathrm{CP}$ cause and $27 \%$ perceived cause of CP as destiny. This is line with the study of Karande et al conducted on parents of children newly diagnosed with CP, about $80.8 \%$ of the respondents are not aware of the cause of the condition before educational intervention [1], another study conducted by Arora, et al 2014 on parents of children with CP also indicated low or lack knowledge of $\mathrm{CP}$ before the educational intervention in about $74.6 \%$ of the participants [9]. After the educational intervention all respondents (100\%) attributed the cause of CP to pregnancy abnormalities, this is similar to the study of Karande et al that showed improvement of the knowledge of participants on the cause of cerebral palsy, about $76.9 \%$ of the participants could correctly explain the cause of cerebral palsy[1]. A study by Arora, et al confirmed our finding that $90.6 \%$ of the participants were able to mention the possible causes of $\mathrm{CP}$ [9].

The knowledge of the participants before educational intervention showed low level of awareness as $67 \%$ of the responses based on the clinical presentation of cerebral palsy was lack of knowledge, this is expected because the participants are pregnant women with non $\mathrm{CP}$ children as such they are not well in contact with the condition enough to know the clinical manifestations. But after the educational intervention the participants' knowledge improved with better responses like difficulty with eating $54.1 \%$, Paralysis 56.8\%, Mental retardation $67.7 \%$

The study also revealed the lack of knowledge for the possible involvements of medical practitioners in the management of CP in the majority of respondents $75.7 \%$ before the educational intervention. A study conducted by Karande et al contradicts the finding of the study that majority of the respondents $69.2 \%$ were able to mention few professionals involved in the management of $\mathrm{CP}[1]$ although the studies differ in respondents as the study involves parents of children with $\mathrm{CP}$ and the respondents in our study are pregnant women with no CP children. But post awareness result showed a lot of improvements to about $88.5 \%$ of the respondent were able to identify more medical practitioners in the management of $\mathrm{CP}$ which is similar to our finding in the study that about $94.6 \%$ and $81.1 \%$ of responses are physiotherapist and paeditricans respectively involved in management of CP.

The pre-awareness knowledge level of the participants on the preventive CP measures is low because about $54.1 \%$ of the participants do not know the measures to be taken in the prevention of this condition but the response improved to about $80 \%$ of responses indicating appropriate measures for the prevention of CP. This finding can be supported with the study of Arora, et al improvements of the participants' knowledge in on CP preventive measures from $20.8 \%$ to $83 \%$ pre and post educational session respectively.

In conclusion, the finding of this study showed low level of CP condition awareness among pregnant women in Kano, Nigeria, but after educational intervention in this study, their knowledge improved and participants were able to give explanations on the cause, clinical manifestation, preventive measures and management, therefore the need for educational programmes on CP during antenatal periods by the medical professionals or through interactive session among the women.

\section{References:}

1. Karande S Patil, Kulkarni, M. Impact of An Educational Program on Parental Knowledge of Cerebral Palsy, Indian J Pediatr, 2008; 75.

2. Fidan F, Baysal O. Epidemiologic Characteristics of Patients with Cerebral Palsy. Open Journal of Therapy and Rehabilitation, 2014; 2: 126-132.

3. Shamsoddini A, Amirsalari S, Hollisaz M, Rahimniya A, Khatibi-Aghda, A. Management of Spasticity in Children with Cerebral Palsy Iran J Pediatr. 2014; 24 (4): 345-51.

4. Hurley DS, et al. Systematic Review of Cerebral Palsy Registries/Surveillance Groups: Relationships between Registry Characteri- 
stics and Knowledge Dissemination Int J Phys Med Rahabil. 2015; 3:2.

5. O'Shea TM. Diagnosis, Treatment, and Prevention of Cerebral Palsy in Near-Term/Term infants Clin Obstet Gynecol. 2008; 51(4): 816-28.

6. Jan MS. Cerebral Palsy: Comprehensive Review and Update, Ann Saudi Med. 2006; 26 (2):123-32.

7. Reddihough DS, Collins, KJ. The epidemiology and causes of cerebral palsy Australian Journal of Physiotherapy, 2003; 49.

8. Dwight J. Rouse et al. A Randomized, Controlled Trial of Magnesium Sulfate for the Prevention of Cerebral Palsy The New England Journal of Medicine. 2008; 359(9):895905.

9. Arora, S K Aggarwal A, Mittal, H. Int J Pediatr. 2014.

10. Borzoo S Nickbakht, M Jalalian M. Effect of Child's Cerebral Palsy on the Mother: a Case Control Study in Ahvaz, Iran Scientific Journal of the Faculty of Medicine in Niš. 2014; 31(1):75-9.

11. Katz RT. Life expectancy for children with cerebral palsy and mental retardation: Implications for life care planning NeuroRehabilitation. 2003; 18: 261-70.

12. ÓNeil ME Palisano, RJ, Westcott, S L. Relationship of therapists' Attitude, Children's Motor Ability, and Parenting Stress to Mothers' perceptions of therapists' behavior
During Early Intervention Physical Therapy. 2001; 81(8) 1412-24.

13. Novak I. Evidence-Based Diagnosis, Health Care, and Rehabilitation for Children with Cerebral Palsy J. Child Neurol. 2014; 29(8) 1141-56.

14. Palit A, Chatterjee AK. Parent-to parent counseling - a gateway for developing positive mental health for the parents of children that have cerebral palsy with multiple disabilities Int J Rehabil Res. 2006; 29(4); 281-8.

15. Zissermann L. Sex of a Parent and knowledge about cerebral Palsy Am J Occup Ther. 1978; 32(80): 500-4.

16. Ribeiro MFM, Barbosa MA, Porto CC. Cerebral palsy and Down syndrome: Level of parental knowledge and information Ciência \& Saúde Coletiva, 2011; 16(4):2099-106.

17. Lewicka M, Wdowiak A, Sulima M, Bakalczuk G, Niesciór B, Ilzecka J. Knowledge of factors predisposing to the occurrence of cerebral palsy among pregnant women. J Pre-Clin Clin Res. 2012; 6(2): 126-30.

\section{Corresponding author:}

Auwal Abdullahi

Email: aabdullahi.pth@buk.edu.ng; therapistauwal@yahoo.com 